\title{
Predictability of Extreme Waves in the Lorenz-96 Model Near Intermittency and Quasi-Periodicity
}

\author{
A. E. Sterk and D. L. van Kekem \\ Johann Bernoulli Institute for Mathematics and Computer Science, University of Groningen, P.O. Box 407, \\ 9700 AK Groningen, Netherlands
}

Correspondence should be addressed to A. E. Sterk; a.e.sterk@rug.nl

Received 14 May 2017; Revised 11 July 2017; Accepted 16 August 2017; Published 24 September 2017

Academic Editor: Davide Faranda

Copyright (C) 2017 A. E. Sterk and D. L. van Kekem. This is an open access article distributed under the Creative Commons Attribution License, which permits unrestricted use, distribution, and reproduction in any medium, provided the original work is properly cited.

\begin{abstract}
We introduce a method for quantifying the predictability of the event that the evolution of a deterministic dynamical system enters a specific subset of state space at a given lead time. The main idea is to study the distribution of finite-time growth rates of errors in initial conditions along the attractor of the system. The predictability of an event is measured by comparing error growth rates for initial conditions leading to that event with all possible growth rates. We illustrate the method by studying the predictability of extreme amplitudes of traveling waves in the Lorenz-96 model. Our numerical experiments show that the predictability of extremes is affected by several routes to chaos in a different way. In a scenario involving intermittency due to a periodic attractor disappearing through a saddle-node bifurcation we find that extremes become better predictable as the intensity of the event increases. However, in a similar intermittency scenario involving the disappearance of a 2-torus attractor we find that extremes are just as predictable as nonextremes. Finally, we study a scenario which involves a 3-torus attractor in which case the predictability of extremes depends nonmonotonically on the prediction lead time.
\end{abstract}

\section{Introduction}

Classical extreme value statistics is concerned with the asymptotic distribution of large values in time series of random variables. The theory, which is based on the extreme value and generalized Pareto distributions, is well developed for stochastic processes both with and without serial dependence; see the text books [1-7]. A recent development is the application of extreme value statistics in the setting of deterministic dynamical systems. The main idea is to evaluate a scalar observable along the evolution of a system and to study under which conditions the same extreme value laws hold as in the case of stochastic processes. Geophysical applications, in which dynamical systems arise as models and observables are physical quantities like wind speed or temperature, form an important motivation for the development of the theory. Very recently, Lucarini et al. [8] published the first text book on extremes in dynamical systems which gives an excellent overview of the latest developments and also provides an extensive source of references.
Statistics only describe the behaviour of extremes over long periods of time. However, for the development of earlywarning systems and risk mitigation strategies the short-term predictability of extremes is of great importance. This leads to the following question: how predictable are extremes? Bodai [9] summarizes three different conclusions that can be found in the literature:

(1) Extremes are better predictable.

(2) Extremes are less predictable.

(3) Extremes can be better or less predictable depending on several factors.

The first conclusion is supported by the work of Hallerberg et al. [10] who studied the predictability of extreme increments in first-order autoregressive process, wind speed recordings, and long-range correlated autoregressive moving averages. In all their examples extremes become better predictable with increasing event size. The results in [11] showed that in i.i.d. stochastic processes large increments are better predictable 
if the process is Gaussian, whereas large increments become less predictable if the underlying distribution has a power law tail. However, in the follow-up study [12], which is concerned with threshold crossings instead of increments, it was found again that extremes are always better predictable. The first conclusion is also supported by the work of Franzke $[13,14]$ in the context of dynamic-stochastic models. Bodai [9] argues that in dynamical systems stronger predictability of extremes may be typical but not universal. The third conclusion is supported by the work of Sterk et al. In [15] it was pointed out that the predictability of extreme values in dynamical systems depends on the observable, the attractor of the system, and the prediction lead time. In [16] it was shown how the tail of the distribution of wind speeds affects their predictability at high thresholds.

The predictability of extremes can be measured in different ways. By treating extreme events as binary events one can measure prediction skill by means of a receiver operator characteristic (ROC) curve which is a graph of the hit rate against the false alarm rate [9-14]. Another possible measure is the extreme dependency score developed by Stephenson et al. [17], which does not tend to zero for vanishingly rare events unlike scores such as the equitable threat score. Alternatively, when predictions are made using a dynamical model, predictability can be measured in terms of the growth rate of errors in the initial condition. The earliest studies on predictability in atmospheric models [18-20] computed the time needed for small errors in the initial condition to double in magnitude. This idea connects with traditional predictability measures for dynamical systems, such as Lyapunov exponents. The latter are asymptotic quantities that are computed for time tending to infinity, which also implies that they are independent of the initial condition [21]. Finitetime Lyapunov exponents and singular values measure the growth rate of errors over a finite time and typically they strongly depend on the initial condition and the prediction lead time. Measures of this type have been developed in celestial mechanics to separate chaotic from regular dynamics $[22,23]$, and they have been used to measure the growth of errors due to model perturbations [24] and the predictability of extremes [15].

Several papers demonstrated that finite-time error growth rates can show large fluctuations along the attractor of the system [25-31]. Benzi and Carnevale [32] argued that a ratio of the average growth rate to the most probable growth rate much smaller than 1 is an indication of enhanced predictability, which means that some events may be better predictable than others. A natural question is then what kind of dynamics can lead to enhanced predictability? For example, in dynamical systems with intermittency the dynamics switches between two or more different dynamical regimes and each regime can be associated with different predictability characteristics. The work in $[33,34]$ shows that in intermittent dynamical systems distributions of finite-time Lyapunov exponents are non-Gaussian and asymmetric and have heavy tails. Hence, in intermittent systems one can expect that some events are better predictable than others.

The aim of this paper is to demonstrate that the predictability of extremes depends on the dynamical regime of the model that is used for the predictions. In particular, we show that in weakly chaotic regimes of a dynamical system the predictability of extremes does not have universal properties. The main idea, which is in the spirit of [32], is to study the distribution of finite-time growth rates of errors in initial conditions along the attractor of the system. Comparing error growth rates of initial conditions leading to an event with all possible growth rates then gives a measure of the predictability of the event. We illustrate the method using the Lorenz-96 model [35]. On the one hand this model is simple enough for performing detailed numerical explorations. On the other hand the model has many dynamical features that are shared by a large class of geophysical models. The Lorenz-96 model can be interpreted as a model for traveling waves. The routes to chaos are myriad and different kinds of attractors can be found [36]. The bifurcation scenarios in the Lorenz-96 model can also be found in more complex geophysical models, such as the atmospheric and oceanic models studied in [37, 38]. We will focus in particular on predictability in the vicinity of bifurcations leading to intermittent and quasi-periodic dynamics.

The remainder of this paper is structured as follows. In Section 2 we explain how to quantify the predictability of an event in a general dynamical system. In Section 3 we introduce the Lorenz-96 model which we will use for our numerical experiments. For three values of the dimension of the model we investigate how the predictability of extreme waves in the model depends on intermittent or quasi-periodic nature of the dynamics. Section 4 concludes the paper with a summary and discussion of the results and suggestions for further research.

\section{Predictability of Dynamical Systems}

This section explains the methodology of quantifying the predictability of an event in a dynamical system. In general, a deterministic dynamical system can be defined as a triple $(M, T, \Phi)$ which consists of a state space $M$, a time set $T$, and an evolution operator $\Phi: T \times M \rightarrow M$, such that the following properties are satisfied:

(i) $T \subset \mathbb{R}$ is an additive half group: $0 \in T$ and for all $t, s \in T$ also $t+s \in T$.

(ii) For all $x \in M$ and $t, s \in T$ we have

$$
\begin{gathered}
\Phi(x, 0)=x \\
\Phi(\Phi(x, t), s)=\Phi(x, t+s) .
\end{gathered}
$$

We also write $\Phi_{t}(x)=\Phi(x, t)$. Particular examples that are included in this setting are discrete time systems, such as iterated maps, and continuous-time systems, such as flows of differential equations; see $[39,40]$. In this paper we assume that the state space is a subset of the Euclidean space, but more generally $M$ can be a Riemannian manifold or a function space.

The predictability of a dynamical system is often quantified in terms of the growth rate of errors in the initial 
condition. Suppose that the initial condition $x_{0} \in M$ is perturbed in the direction of $v$; then $\left\|\Phi_{\tau}\left(x_{0}+\epsilon v\right)-\Phi_{\tau}\left(x_{0}\right)\right\| / \epsilon$ is the error growth rate over a time interval of length $\tau$. Harle et al. [28] studied the statistics of these growth rates and their dependence on the parameters $\epsilon$ and $\tau$ in the setting of 2 -dimensional dissipative and conservative maps. The error growth was found to increase exponentially fast with $\tau$ when $\tau$ is small. For larger values of $\tau$ the error growth follows a power law which depends on the magnitude of $\epsilon$. In their paper it is suggested that these results are quite general.

In this paper we will make the idealized assumption that the initial perturbation size $\epsilon$ is infinitesimally small. Under this assumption the error at time $\tau$ is then given by

$$
\lim _{\epsilon \rightarrow 0} \frac{\Phi_{\tau}\left(x_{0}+\epsilon v\right)-\Phi_{\tau}\left(x_{0}\right)}{\epsilon}=D \Phi_{\tau}\left(x_{0}\right) v,
$$

where the derivative is taken with respect to the initial condition $x_{0}$ in the direction of the vector $v$. The worstcase error growth over a time interval of length $\tau$ can be computed by maximizing the following Rayleigh quotient over all nonzero vectors $v$ :

$$
\frac{\left\|D \Phi_{\tau}\left(x_{0}\right) v\right\|^{2}}{\|v\|^{2}}=\frac{v^{\top} D \Phi_{\tau}\left(x_{0}\right)^{\top} D \Phi_{\tau}\left(x_{0}\right) v}{v^{\top} v},
$$

where $\|\cdot\|$ denotes the Euclidean norm. A standard result in linear algebra [41] implies that the quotient (3) attains a maximum if and only if $v$ is the eigenvector of $D \Phi_{\tau}\left(x_{0}\right)^{\top} D \Phi_{\tau}\left(x_{0}\right)$ corresponding to the largest eigenvalue. Equivalently, the maximum is attained precisely when $v$ is the right singular vector corresponding to the largest singular value of $D \Phi_{\tau}\left(x_{0}\right)$, which throughout this paper will be denoted by $\sigma\left(x_{0}, \tau\right)$. In this way we obtain a measure of finite-time predictability for a given initial condition $x_{0} \in M$.

In many applications it is often important to quantify the predictability of a certain event taking place in the future. We define an event to be a subset $E$ of the state space $M$. For a given initial condition $x_{0} \in M$ we say that the event $E$ occurs at time $\tau$ if $\Phi_{\tau}\left(x_{0}\right) \in E$, or, equivalently, $x_{0} \in$ $\Phi_{\tau}^{-1}(E)$. The predictability of the event $E$ can be quantified as follows. Assume that the dynamical system $(M, T, \Phi)$ is equipped with an invariant probability measure $\mu$ supported on some attractor $A \subset M$ (in which case we also assume that $E \subset A)$. This means that $\mu(A)=1$ and $\mu\left(\Phi_{t}^{-1}(S)\right)=\mu(S)$ for all measurable subsets $S \subset A$. Then the distribution function of the time- $\tau$ singular values is given by

$$
\mathbb{P}_{\tau}(\sigma \leq s)=\mu(\{x \in A \mid \sigma(x, \tau) \leq s\}) .
$$

The conditional distribution of time- $\tau$ singular values given that the event $E$ occurs at time $\tau$ reads as

$$
\mathbb{P}_{\tau}(\sigma \leq s \mid E)=\frac{\mu\left(\{x \in A \mid \sigma(x, \tau) \leq s\} \cap \Phi_{\tau}^{-1}(E)\right)}{\mu(E)},
$$

where we have used that $\mu$ is an invariant measure so that $\mu\left(\Phi_{\tau}^{-1}(E)\right)=\mu(E)$. The predictability of the event $E$ can be quantified by comparing both distributions. For example, if the right endpoint of $\mathbb{P}_{\tau}(\sigma \leq s \mid E)$ is much smaller than the right end point of $\mathbb{P}_{\tau}(\sigma \leq s)$, then the event $E$ can be called predictable. In the limit $\tau \rightarrow \infty$ all events become equally predictable.

The advantage of the approach outlined in this section is the fact that it combines measures of predictability and the statistical recurrence properties of the system via its invariant measure. For simple dynamical systems for which the growth of errors can be computed analytically and for which the invariant measure is known the distributions (4) and (5) can be computed analytically. Hence, our approach may be used to derive general statements on the predictability of extremes for simple classes of dynamical systems in a rigorous way. This idea will be pursued in forthcoming work. Also note that the methodology applies to arbitrary events. This in particular includes the case of rare events, but these need not be extreme events in which some observable exceeds a threshold.

\section{Results}

3.1. The Lorenz-96 Model. In [35] Lorenz introduced a onedimensional atmospheric model to study fundamental issues regarding the predictability of the atmosphere and weather forecasting. The model can be interpreted as a model for atmospheric waves traveling along a circle of constant latitude. We divide the latitude circle into $n$ equal sectors and define for the $i$-th sector a distinct variable $x_{i}$. The variables $x_{1}, \ldots, x_{n}$ can be interpreted as meteorological quantities, such as pressure or vorticity, where the index $i$ of each variable $x_{i}$ plays the role of longitude. The dynamical equations are

$$
\frac{d x_{i}}{d t}=x_{i-1}\left(x_{i+1}-x_{i-2}\right)-x_{i}+F, \quad i=1, \ldots, n>3,
$$

with the periodic "boundary condition" $x_{i+n}=x_{i}$. The dimension $n \in \mathbb{N}$ and forcing $F \in \mathbb{R}$ are free parameters. The Lorenz-96 model is often used to test data assimilation methods [42, 43] and subgrid scale parameterizations [44], for studies in statistical mechanics [45, 46], and in the general study of spatiotemporal chaos [47]. In this paper we use the Lorenz-96 model to study the predictability of extreme events in the vicinity of bifurcations.

The point $x_{F}=(F, \ldots, F)$ is clearly an equilibrium solution of (6) for all $n \in \mathbb{N}$ and all $F \in \mathbb{R}$. For all $n \geq 4$ this equilibrium becomes unstable through either a supercritical Hopf or a double-Hopf bifurcation for $8 / 9<F<3 / 2$ [36]. In both cases a stable periodic attractor is born which has the physical interpretation of a traveling wave. Figure 1 shows the spatiotemporal properties of these waves: the period and the spatial wave number are plotted as a function of $n$. In [36] it was proved analytically that the period tends to a finite limit as $n \rightarrow \infty$, but the wave number increases monotonically with $n$.

The periodic attractor representing the traveling wave can undergo several subsequent bifurcations, such as period doubling bifurcations or Neimmark-Sacker bifurcations. Further bifurcations lead to strange attractors via a multitude of routes to chaos which depend on the dimension $n$ [36]. Hence, the Lorenz-96 exhibits successive bifurcations of traveling waves. The spatiotemporal properties of the resulting waves 


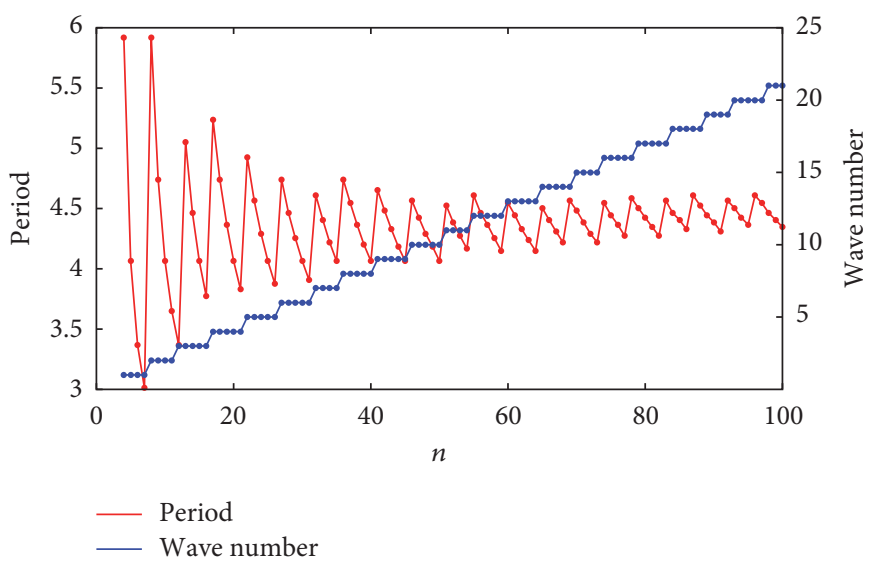

Figure 1: The period and spatial wave number of the traveling wave born at the first Hopf bifurcation of the Lorenz-96 model as a function of the dimension $n$. Note that the period tends to a finite number as $n \rightarrow \infty$, but the wave number is monotonically increasing.

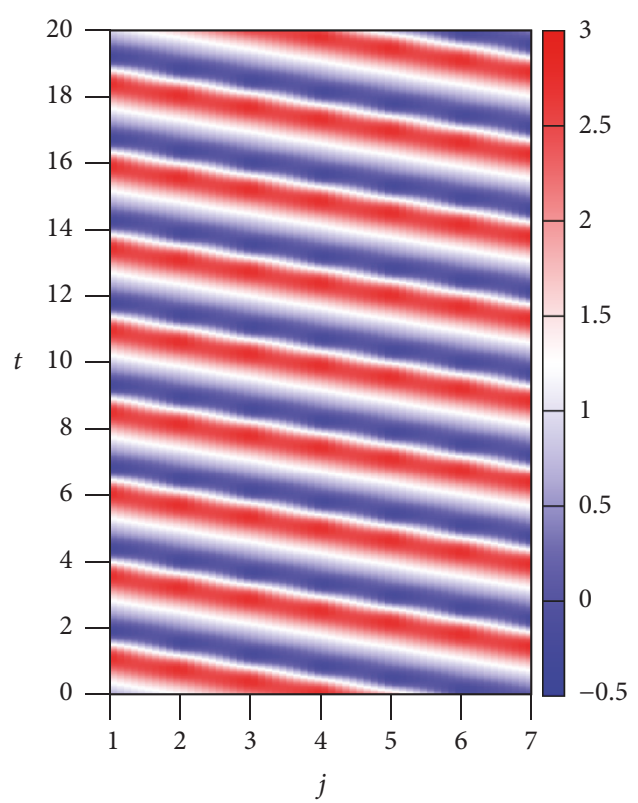

(a)

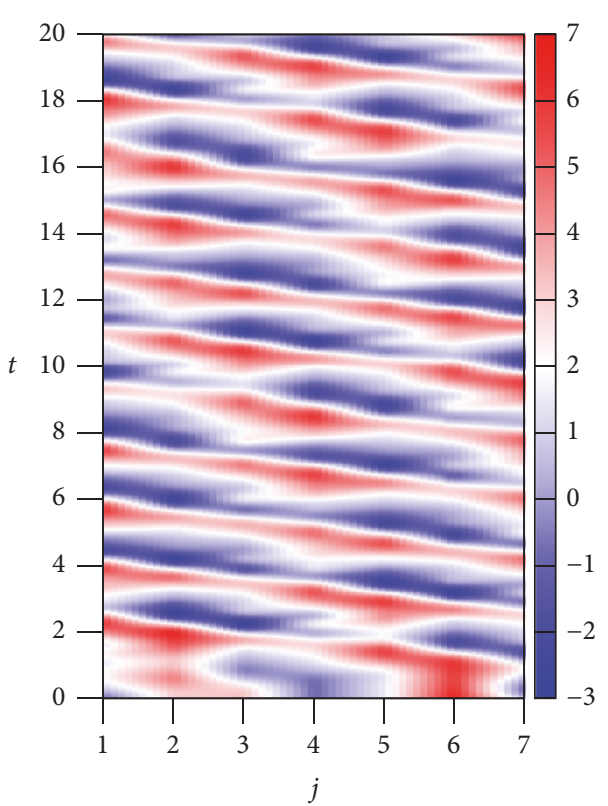

(b)

FIGURE 2: Hovmöller diagrams of two attractors in the Lorenz-96 model for $n=7$ and $F=2$ (a) and $F=4.4$ (b). The value of $x_{j}(t)$ is plotted as a function of $t$ and $j$. For visualization purposes linear interpolation between $x_{j}$ and $x_{j+1}$ has been applied in order to make the diagram continuous in the variable $j$.

are "inherited" from the periodic attractor that was born at the Hopf bifurcation. For an example, see Figure 2 for two traveling waves in dimension $n=7$. A very similar scenario was found in a Galerkin projection of a shallow water model that was used to study the dynamical mechanisms behind atmospheric low-frequency variability [38].

The particular interest of this paper is the predictability of so-called extreme events in which an observable evaluated along an evolution of the system exceeds a threshold. Concrete examples are models for weather and climate in which extremes of physical quantities such as wind speed are of great importance $[15,16,48]$. For the Lorenz-96 model we will study the predictability of events of the form

$$
E_{u}=\left\{x \in \mathbb{R}^{n}: x_{1}>u\right\} .
$$

Hence, if $x(0)$ is an initial condition, then $x(\tau) \in E_{u}$ is the event that the amplitude of the traveling wave measured at the first "grid point" exceeds the threshold $u$ at time $\tau$. For these events the distributions (4) and (5) not only depend on the prediction lead time $\tau$, but also on the event threshold $u$. In this paper we will study how this dependence is influenced by (nearby) bifurcations of the system. Note that due to the 


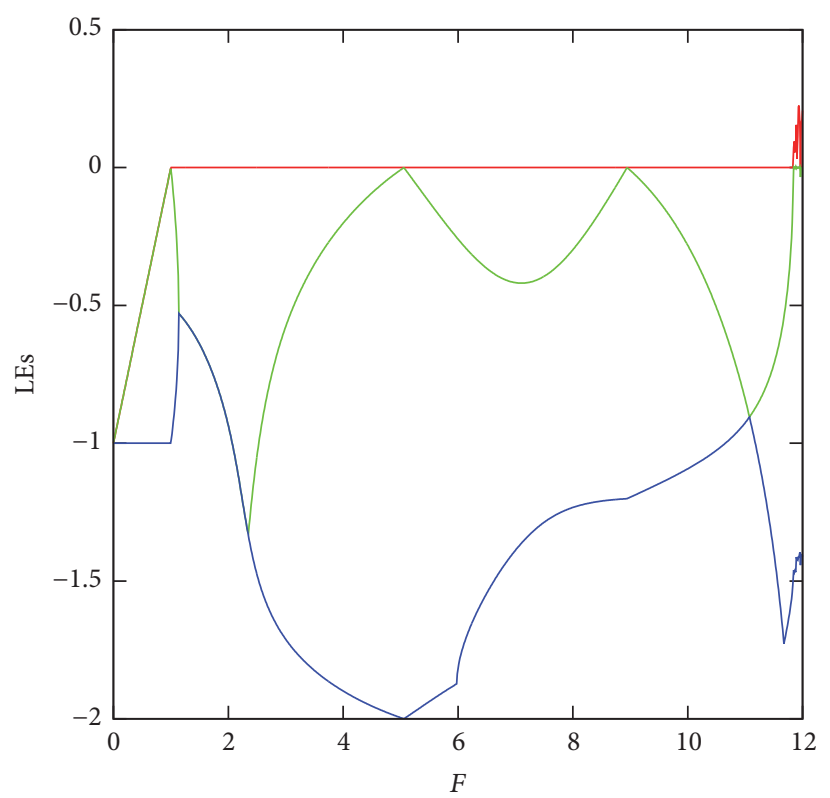

(a)

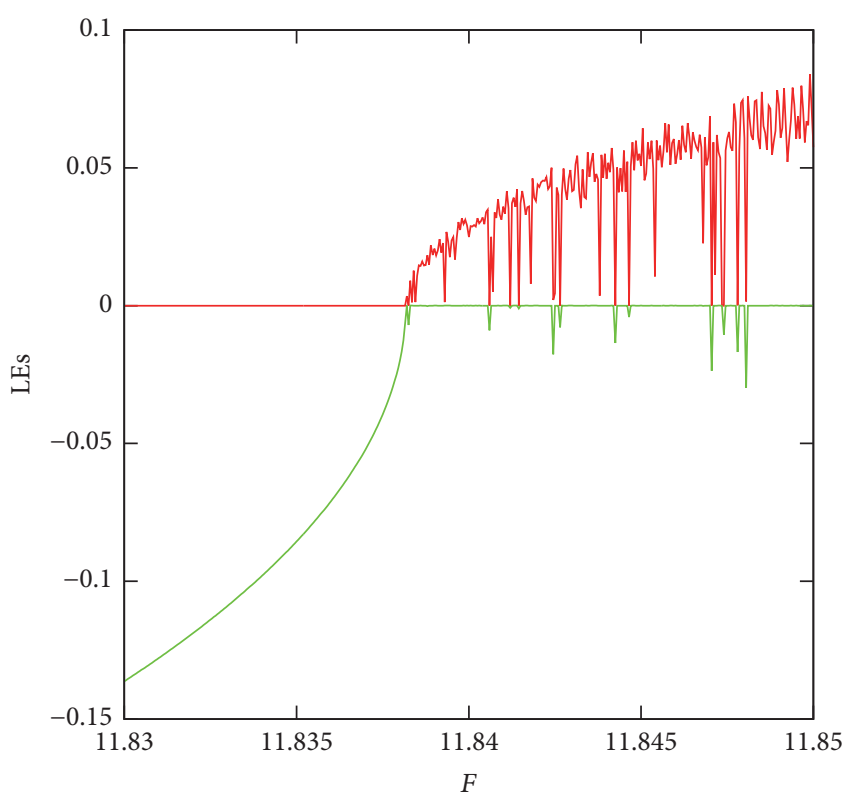

(b)

FIGURE 3: Bifurcation diagrams of attractors in the Lorenz-96 model for $n=4$. The three largest Lyapunov exponents are plotted as a function of the parameter $F$. At $F \approx 11.8382$ a periodic attractor disappears through a saddle-node bifurcation and a chaotic attractor is detected (see the magnification in (b)).

circulant symmetry of the Lorenz-96 model (6) the results obtained for the event $E_{u}$ defined in (7) will not change if the inequality $x_{1}>u$ is replaced by $x_{i}>u$ for any other value of $i=2, \ldots, n$.

For simple dynamical systems for which the invariant measure is known the distributions (4) and (5) can be computed analytically. However, for the Lorenz-96 model they have to be approximated by their empirical counterparts obtained from numerical simulations. In order to provide a good sampling of the attractor of the Lorenz-96 system as far as both local and global fluctuations are concerned we computed the distributions by means of an orbit on the attractor consisting of $10^{5}$ points with a time step of 0.1 . The starting point of the orbit is obtained by a transient integration of 500 time units using a random initial condition.

3.2. Intermittent Periodicity for $n=4$. Figure 3 shows the bifurcation diagram of the Lorenz-96 model for $n=4$. The equilibrium $x_{F}=(F, F, F, F)$ becomes unstable at $F=$ 1 through a supercritical Hopf bifurcation. The periodic attractor remains stable until $F \approx 5.06$ where it exchanges stability with another periodic attractor. However, at $F \approx$ 8.93 the original periodic attractor gains stability again. Finally, at $F_{\mathrm{SN}} \approx 11.8382$, it disappears through a saddlenode bifurcation and a chaotic attractor is detected. Figure 4 shows the periodic attractor and the chaotic attractor just before and after the saddle-node bifurcation. The dynamics on the chaotic attractor consists of alternations between nearly periodic and chaotic behaviour. This is the classical type 1 intermittency scenario described by Pomeau and Manneville [49]. Note that, for intermittency to occur, it is not only necessary to have an attractor that disappears through a bifurcation, but also there has to be a global dynamical mechanism that enables recurrent visits to the location of the formerly existing attractor in state space. In the case of the Lorenz-96 system we have identified a nearby heteroclinic cycle between four equilibria that can provide such a mechanism; see [36] for further details.

Figure 5 shows the mean and the left and right endpoints of the distribution (4) for $\tau=1$ as a function of the parameter $F$. Clearly, the variability of the singular values along the attractor increases very sharply after the saddlenode bifurcation. Note that the largest Lyapunov exponent in the bifurcation diagram of Figure 3 shows a more gradual increase of square root order except for the presence of narrow windows with periodic dynamics. For $F>11.8382$, which belongs to the chaotic regime after the periodic attractor has disappeared, the right endpoint of (4) shows large fluctuations: peaks can differ in magnitude by a factor of 10 or larger. The left endpoint suddenly decreases after the saddle-node bifurcation, which means that the predictability of some events can potentially be enhanced. In particular, this leads to the question whether the predictability of extremes can be enhanced.

Figure 6 shows graphical representations of the distributions (4) (in black) and (5) (in color) in the form of box plots for the prediction lead times $\tau=0.5, \ldots, 5$. The support of the unconditional probability distribution (4) becomes larger as the lead time $\tau$ increases. More specifically, consider the right endpoint $\sigma_{\infty}$ of the distribution which is defined as the largest singular value of the sample and which is a measure for worst-case predictability. A least squares fit computed over the lead times $\tau=0.25,0.5, \ldots, 1.5$ (not all shown in Figure 6) 


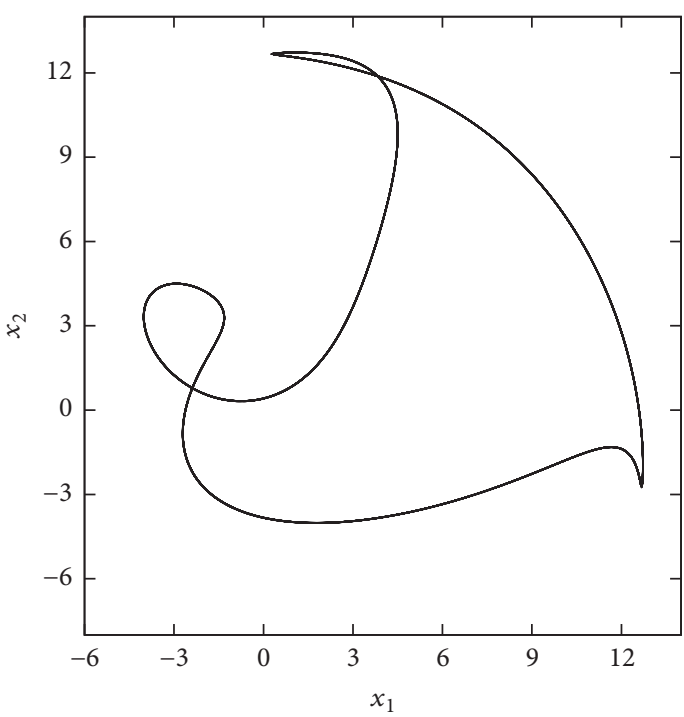

(a)

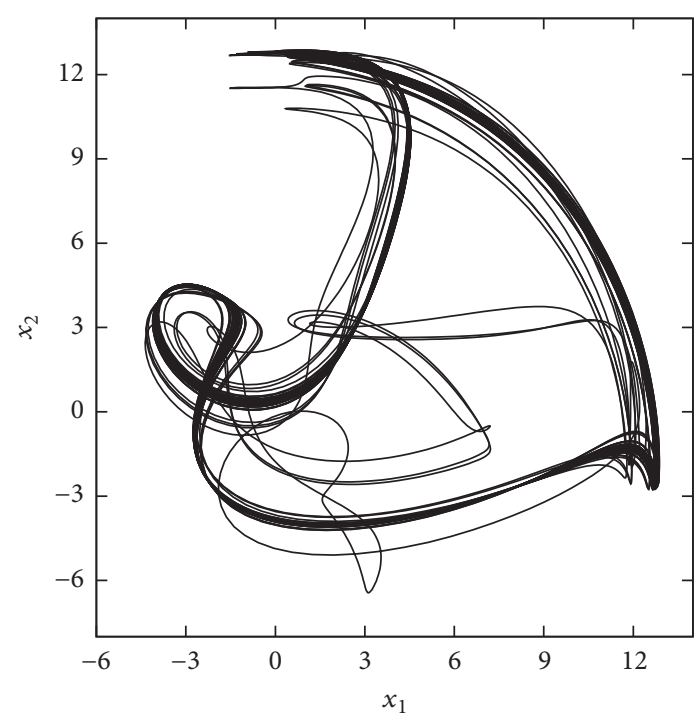

(b)

Figure 4: Periodic attractor of the Lorenz-96 model for $n=4$ and $F=11.83$ (a) and a chaotic attractor for $F=11.85$ that appears after the periodic attractor disappears through a saddle-node bifurcation (b).

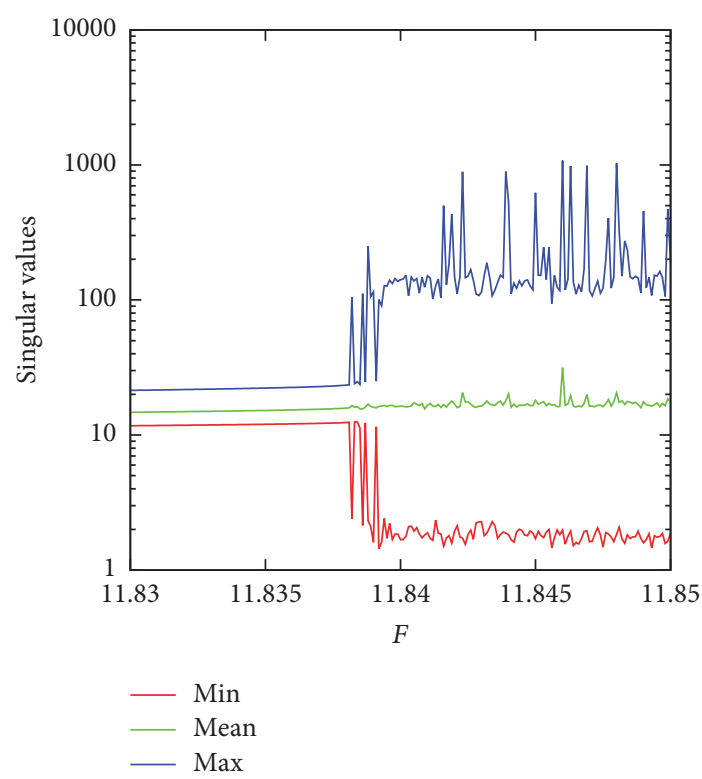

FIgURE 5: The left endpoint, mean, and right endpoint of the distribution (4) plotted as a function of the parameter $F$ for $n=4$ and predictability lead time $\tau=1$. The grid on the horizontal axis consists of 200 points; for each value of $F$ the distribution (4) is approximated by computing singular values along an orbit of $10^{3}$ points.

gives $\sigma_{\infty}=O\left(e^{6.61 \tau}\right)$ which shows that up to $\tau=1.5$ the right end point $\sigma_{\infty}$ increases exponentially with the lead time. For larger lead times, however, $\sigma_{\infty}$ tends to a constant. The exponential growth of $\sigma_{\infty}$ for short lead times has been found earlier in the low-dimensional systems used in the study by Harle et al. [28] who also point out that for finite-size initial errors a power law behaviour will be observed.
Figure 6 also shows that the right endpoint of the conditional distribution (5) grows substantially slower than that of the unconditional distribution (4) for lead times $\tau>0.5$. Figure 7 shows how the conditional distributions change as a function of the threshold $u$ for fixed lead times. For $\tau=$ 0.2 the interquartile range shifts towards the left endpoint, whereas the right end point is nearly constant. However, for $\tau=1.2$ the right endpoint decreases exponentially fast with the threshold quantile $q$ : a linear fit gives that $\sigma_{\infty}=O\left(e^{-26.1 q}\right)$. The main conclusion drawn from these observations is that for $\tau \leq 1.5$ the worst-case error growth, as represented by the right endpoint of the distribution (4), increases exponentially with the prediction lead time $\tau$, but errors for extreme events grow at a much slower rate. For $\tau>1.5$ the right endpoint $\sigma_{\infty}$ of (4) remains nearly constant, but also in this case the right endpoint for the conditional distribution (5) remains several orders of magnitude smaller.

Figure 8 shows a time series of the Lorenz-96 model. Note that $x_{1}(t+\tau)$ is plotted as a function of $t$ with $\tau=1.2$ rather than $x_{1}(t)$ itself. By plotting $\sigma(x(t), \tau)$ in the same figure we can study whether initial conditions leading to extremes typically have small or large error growth rates and whether this is related to particular features of the dynamics. The time series clearly shows alternations of periodic and aperiodic dynamics, which is characteristic for type 1 intermittency. During intervals with periodic dynamics the singular values have a magnitude of $O(10)$. However, during interruptions of periodicity (which in Figure 8 are visible near $t=275$ and $t=315)$ the singular values show very large spikes with typical magnitudes of $O\left(10^{2}\right)$ and $O\left(10^{3}\right)$. This explains why the box plots in Figure 6 exhibit long tails. Note that in $[33,34]$ exponential tails have also been found for finite-time Lyapunov exponents near intermittent dynamics of the logistic map. Also note that in the intervals of 


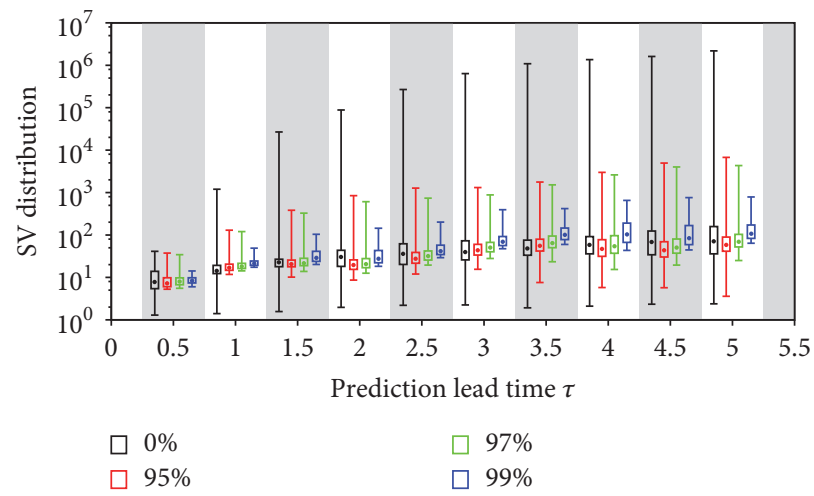

(a)
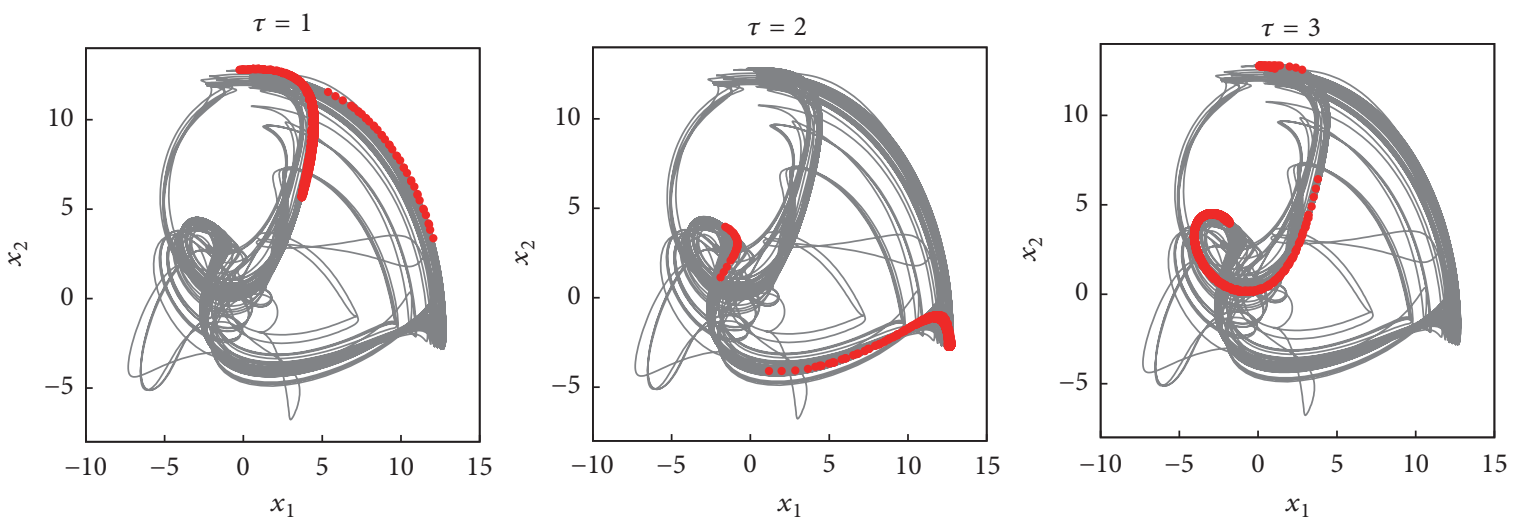

(b)

Figure 6: Box plots of distributions of singular values plotted as a function of the prediction lead time $\tau$ for $n=4$ and $F=11.85$. The black box plots represent the unconditional distribution (4). The colored box plots represent the conditional distribution (5) for the event $E_{u}$ where the threshold $u$ is chosen as the 95th, 97th, or 99th percentile. (b) shows initial conditions (red points) on the attractor for which the event $E_{u}$ takes place at time $\tau=1,2,3$ where $u$ is the 99 th percentile.

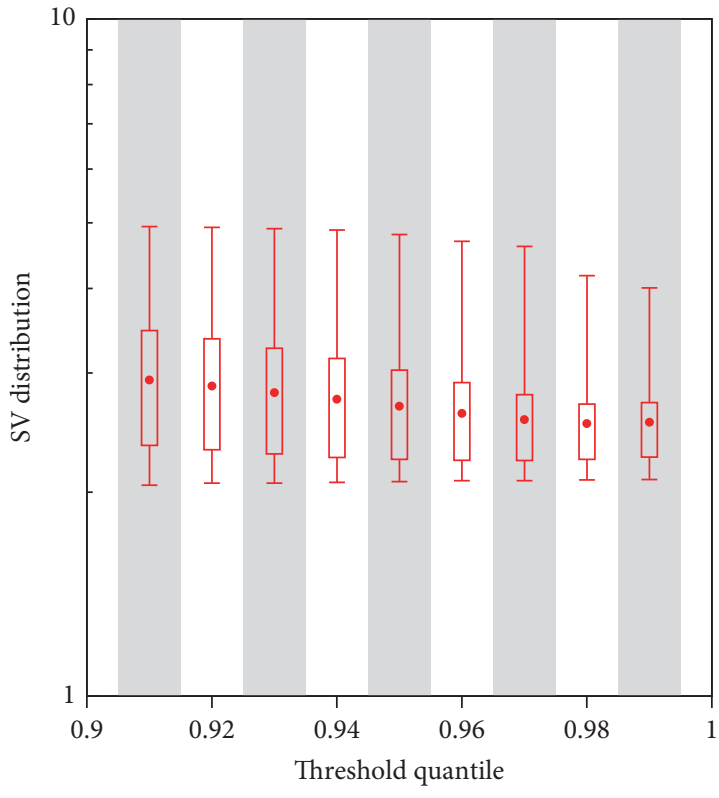

(a)

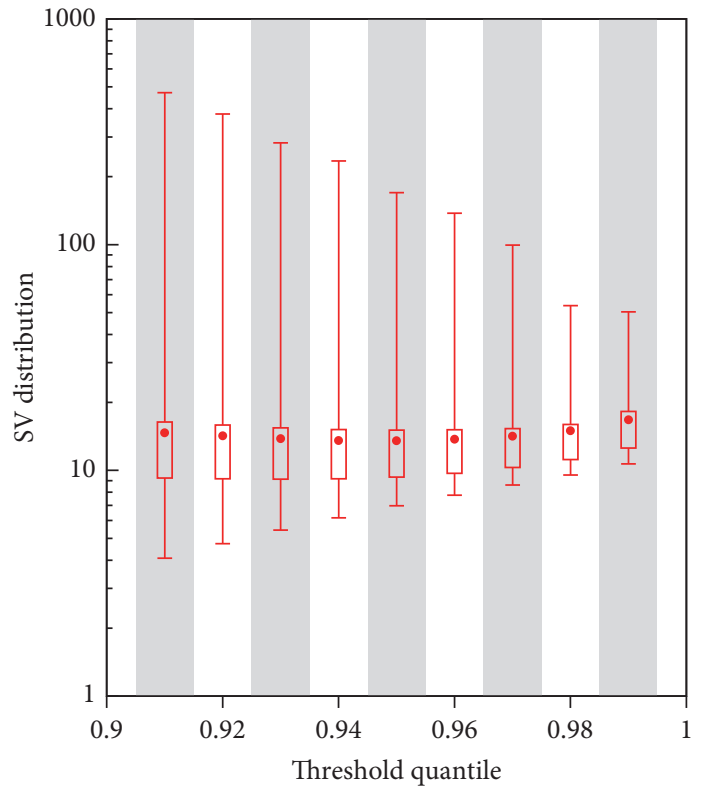

(b)

Figure 7: Box plots of the conditional distribution of singular values (5) for the event $E_{u}$ as a function of the threshold quantile for $n=4$ and $F=11.85$. (a) $\tau=0.2$. (b) $\tau=1.2$. 


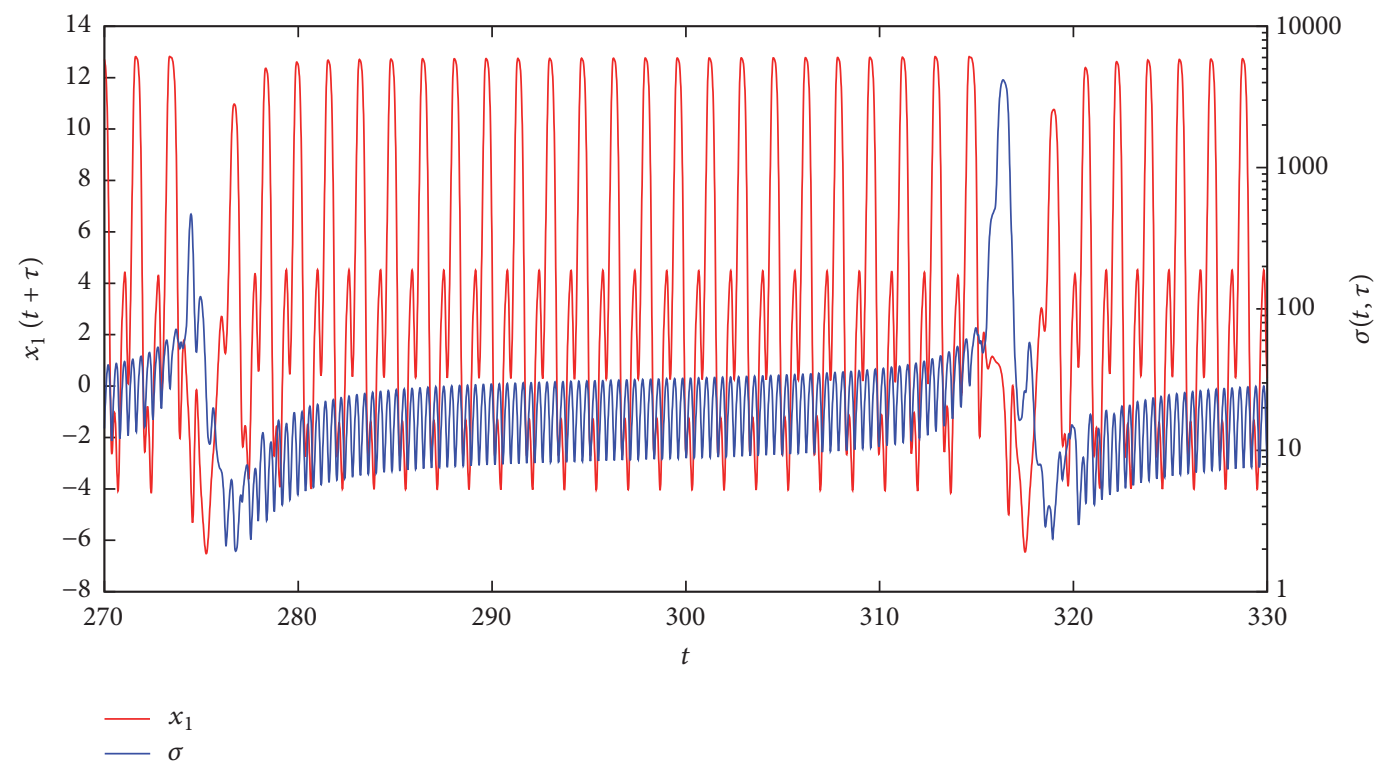

FIGURE 8: Time series of the Lorenz-96 model for $n=4$ and $F=11.85$ in which $x_{1}(t+\tau)$ is plotted together with $\sigma(t, \tau)$ for $\tau=1.2$. The plot illustrates that initial conditions leading to the event $E_{u}$ at time $\tau$ have systematically a low error growth rate $\sigma$.

TABLE 1: The exponential growth of the right endpoint of (4) with lead time for $n=4$ and different parameter values of the parameter $F$.

\begin{tabular}{cccccccc}
\hline$F$ & 11.85 & 11.90 & 11.95 & 12.00 & 12.05 & 12.10 & 12.15 \\
\hline$\sigma_{\infty}$ & $O\left(e^{6.61 \tau}\right)$ & $O\left(e^{6.31 \tau}\right)$ & $O\left(e^{6.28 \tau}\right)$ & $O\left(e^{6.71 \tau}\right)$ & $O\left(e^{6.47 \tau}\right)$ & $O\left(e^{2.55 \tau}\right)$ & $O\left(e^{6.48 \tau}\right)$ \\
\hline
\end{tabular}

aperiodic behaviour $x_{1}$ does not reach extreme values. These observations explain why in Figure 7 the right endpoint of the distribution decreases with the event threshold.

The computations by Pomeau and Manneville [49] suggest that in the type 1 intermittency scenario the maximal Lyapunov exponent grows like $O\left(\sqrt{F-F_{\mathrm{SN}}}\right)$ where $F_{\mathrm{SN}} \approx$ 11.8382 is the parameter value of the saddle-node bifurcation. Such behaviour is indeed visible in Figure 3 with the exception of the presence of narrow windows with periodic dynamics. This suggests that further away from the bifurcation the system becomes more chaotic. A natural question then is how will the predictability of extremes behave further away from the saddle-node bifurcation? Table 1 shows the exponential growth of $\sigma_{\infty}$ for $0<\tau \leq 1.5$ for different parameter values $F$. In each case a least squares fit computed over the lead times $\tau=0.25,0.5, \ldots, 1.5$ has been used. The behaviour is rather stable with $F$ with the exception of $F=12.1$ in which case a stable periodic attractor appears amidst the chaotic regime. Figure 9 shows a similar diagram as in Figure 6 but for the parameter value $F=12.15$ which is further away from the saddle-node bifurcation. These results suggest that the predictability of the event $E_{u}$ is rather stable across a broad range of parameter values for $F$, except for periodic windows within the chaotic regime.

3.3. Intermittent Quasi-Periodicity for $n=7$. Figure 10 shows the bifurcation diagram of the Lorenz-96 model for dimension $n=7$. The equilibrium $x_{F}=(F, \ldots, F)$ becomes unstable at $F \approx 1.182$ through a supercritical Hopf bifurcation. The periodic attractor remains stable until $F \approx 2.717$ where it

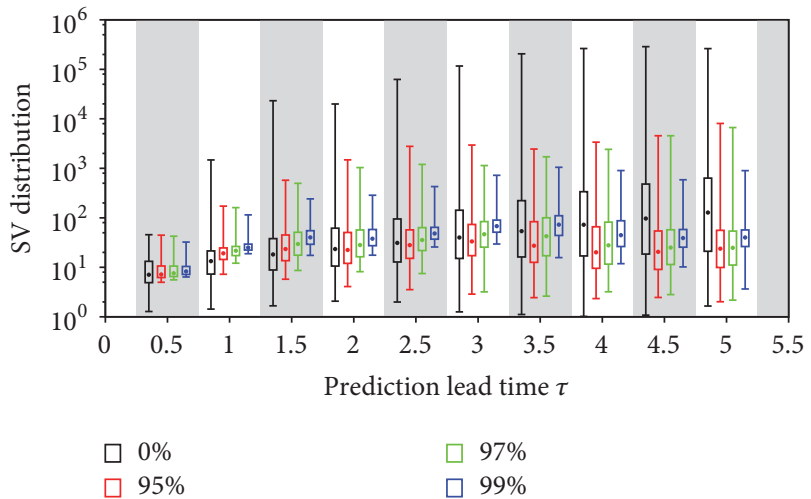

FIGURE 9: As Figure 6, but for the parameter value $F=12.15$ which is further away from the saddle-node bifurcation.

bifurcates through a Neimmark-Sacker bifurcation. The resulting 2-torus attractor remains stable until $F \approx 4.272$ where it disappears through a quasi-periodic saddle-node bifurcation $[40,50]$. Figure 11 shows a Poincaré section of the quasiperiodic attractor before the bifurcation and the chaotic attractor just after the bifurcation. The trace of the formerly existing 2-torus attractor is clearly visible. The dynamics is characterized by alternations between quasi-periodic and chaotic dynamics. This is a form of intermittency but of a different nature than type 2 intermittency described by Pomeau and Manneville [49] since the latter scenario involves the disappearance of a stable periodic orbit instead of a 2torus attractor. 


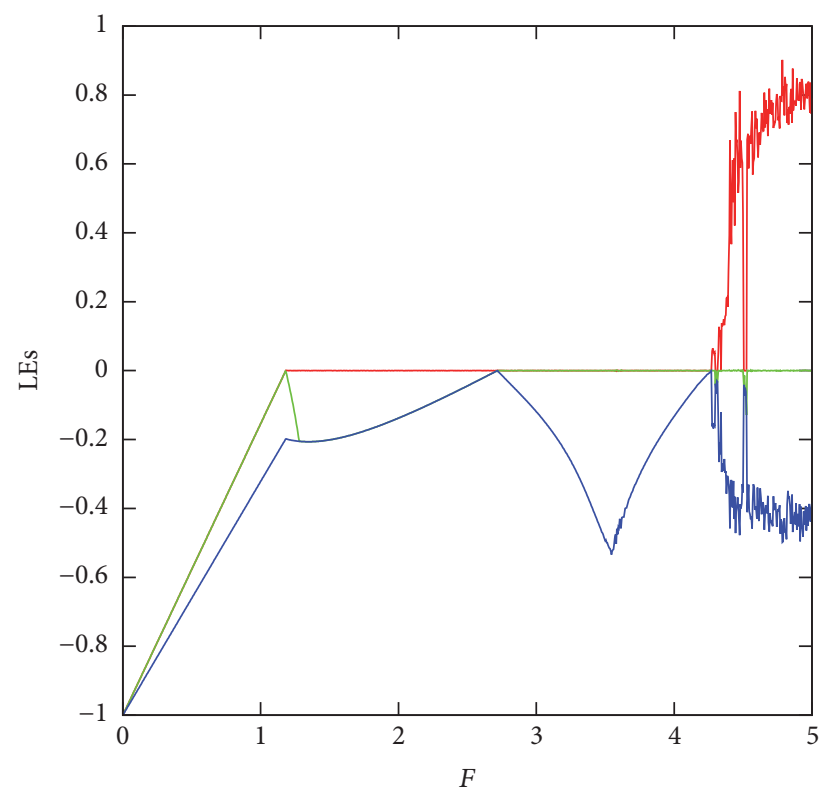

(a)

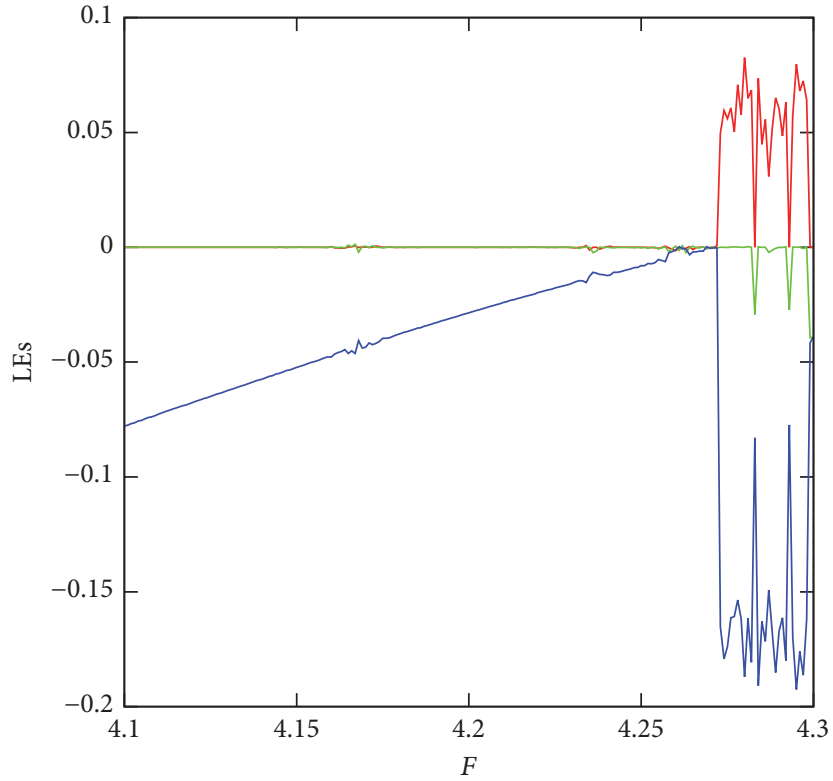

(b)

Figure 10: As Figure 3, but for dimension $n=7$. In this case a 2-torus attractor disappears through a quasi-periodic saddle-node bifurcation which leads to a chaotic attractor.

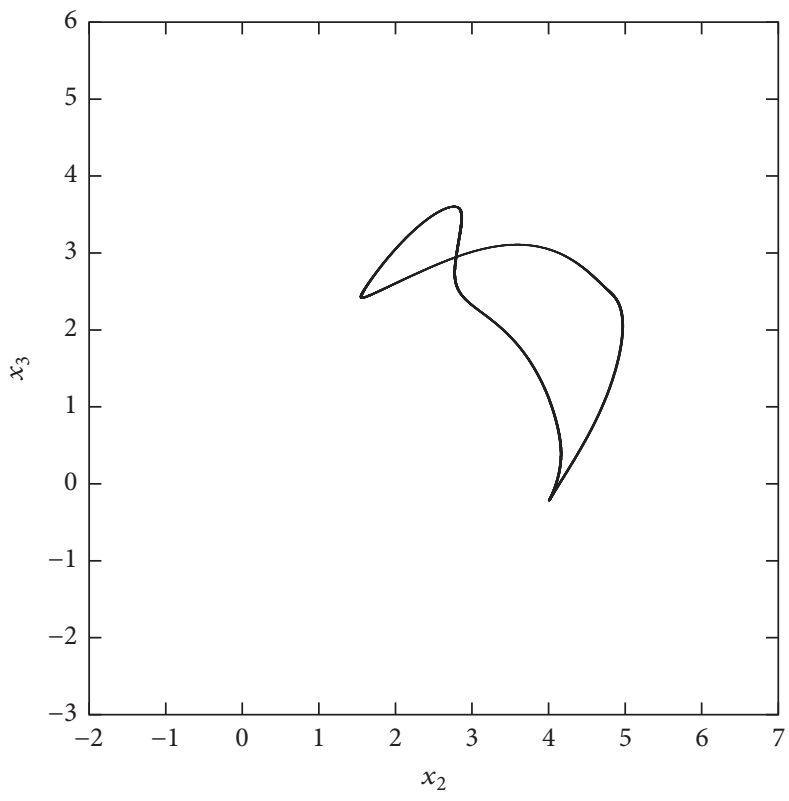

(a)

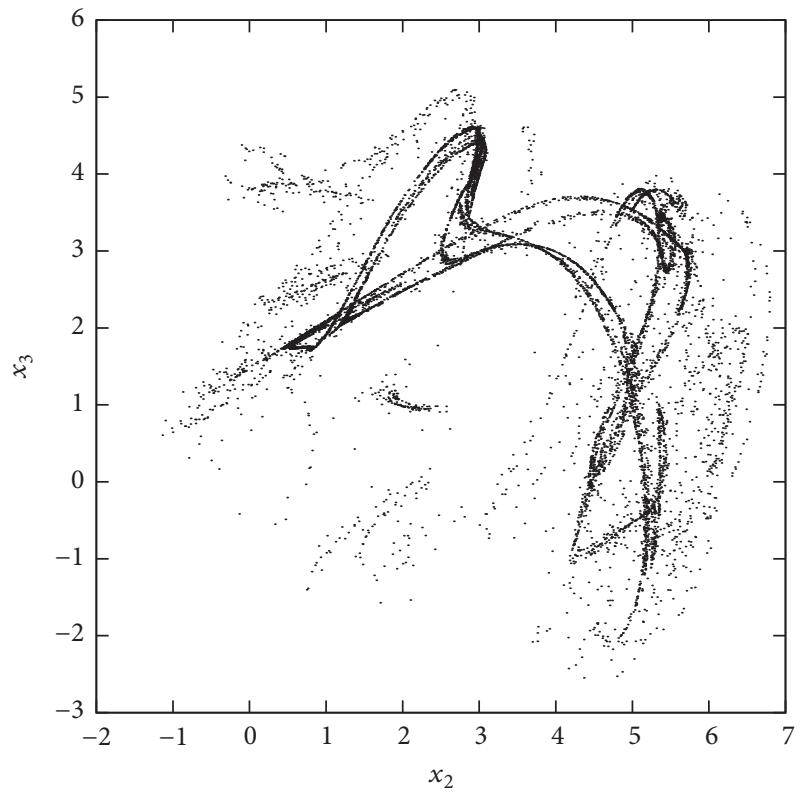

(b)

Figure 11: A quasi-periodic attractor of the Lorenz-96 model for $n=7$ and $F=4.0$ (a) and a chaotic attractor for $F=4.4$ (b) plotted in the Poincaré section $x_{1}=2.5$.

In this case the box plots in Figure 12 indicate that predictability of the event $E_{u}$ does not increase with the threshold $u$. The right end points of both distributions (4) and (5) grow approximately like $\sigma_{\infty}=O\left(e^{1.60 \tau}\right)$ as a function of $\tau$. Also note that the interquartile range of (5) shifts towards larger values as $u$ increases. Table 2 shows that further away from the quasi-periodic saddle-node bifurcation (i.e., for larger $F$ ) the right endpoint $\sigma_{\infty}$ of both the distributions (4) and (5) grows faster with $\tau$. For the parameter values in Table 2 the corresponding box plots are qualitatively similar to Figure 12 


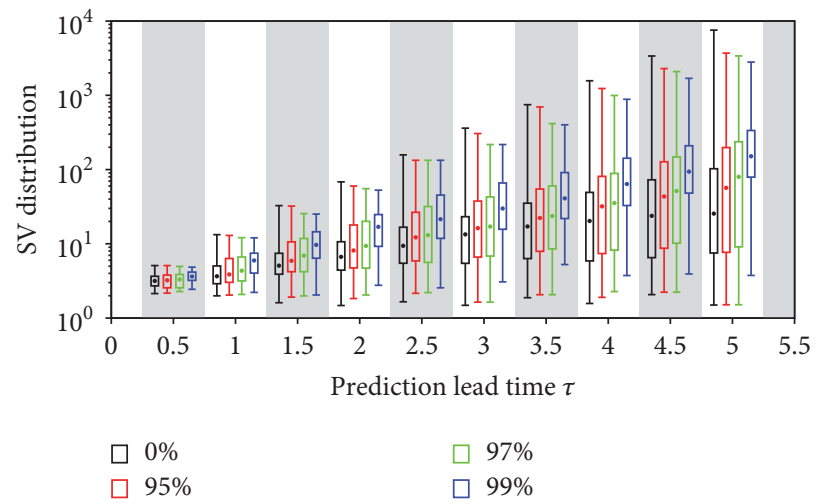

(a)
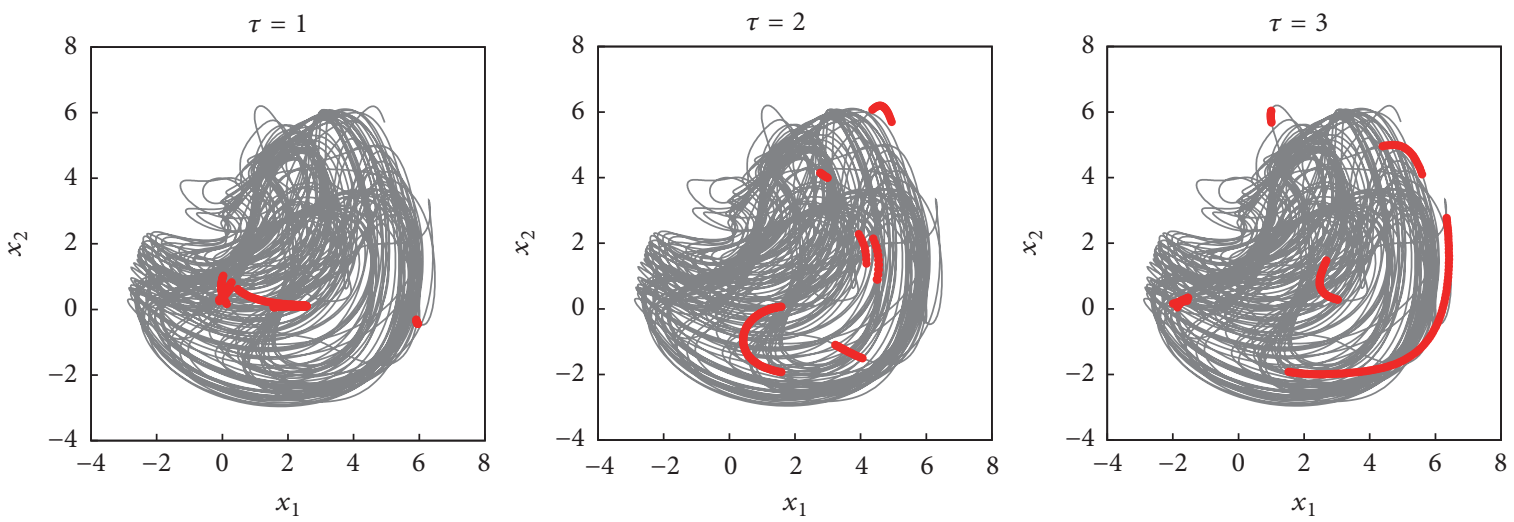

(b)

FIGURE 12: As Figure 6, but for $n=7$ and $F=4.4$.

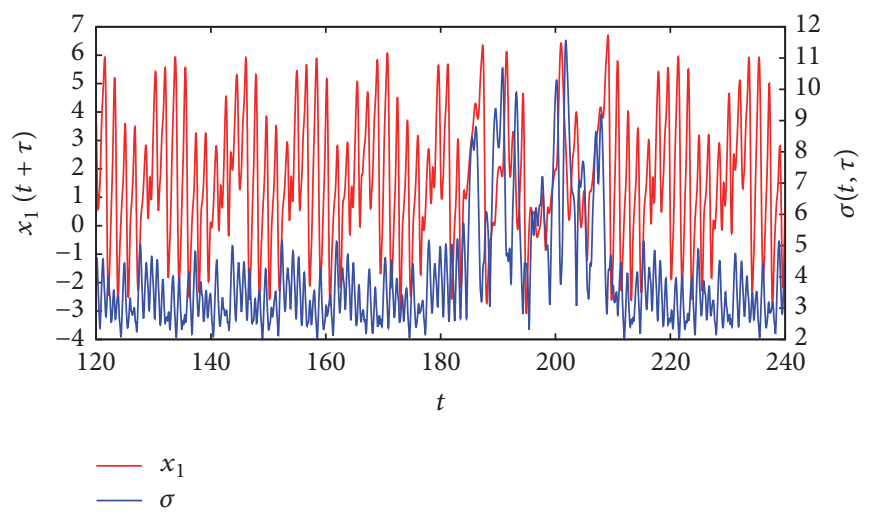

Figure 13: As Figure 8, but for $n=7, F=4.4$, and $\tau=1$. The plot illustrates that initial conditions leading to the event $E_{u}$ at time $\tau$ can have both large and small error growth rates $\sigma$.

and therefore they are not shown. These observations imply that initial conditions that lead to the extreme event $E_{u}$ are typically associated with large error growth rates.

The question is how can the unpredictability of extremes be explained in terms of the intermittent dynamics? Figure 13 shows a time series in which $x_{1}(t+\tau)$ and $\sigma(x(t), \tau)$ are plotted as a function of $t$ for $\tau=1$ fixed. The time series clearly shows an episode of quasi-periodic dynamics which is interrupted for $t \in[180,210]$. During intervals of quasi-periodic dynamics the singular values have magnitudes ranging between 2 and 5. The 2-torus attractor at $F=4$ has singular values in the same range. During intervals of chaotic dynamics the singular values are typically much larger and have a magnitude ranging between 2 and 12. Also note that $x_{1}(t)$ attains extreme values in both the quasi-periodic and the chaotic regime. These observations explain why 
TABLE 2: The exponential growth of the right endpoint of (4) with lead time for $n=7$ and different parameter values of the parameter $F$.

\begin{tabular}{cccccccc}
\hline$F$ & 4.4 & 4.5 & 4.6 & 4.7 & 4.8 & 4.9 & 5.0 \\
\hline$\sigma_{\infty}$ & $O\left(e^{1.60 \tau}\right)$ & $O\left(e^{1.72 \tau}\right)$ & $O\left(e^{1.82 \tau}\right)$ & $O\left(e^{1.90 \tau}\right)$ & $O\left(e^{2.01 \tau}\right)$ & $O\left(e^{2.00 \tau}\right)$ & $O\left(e^{2.14 \tau}\right)$ \\
\hline
\end{tabular}

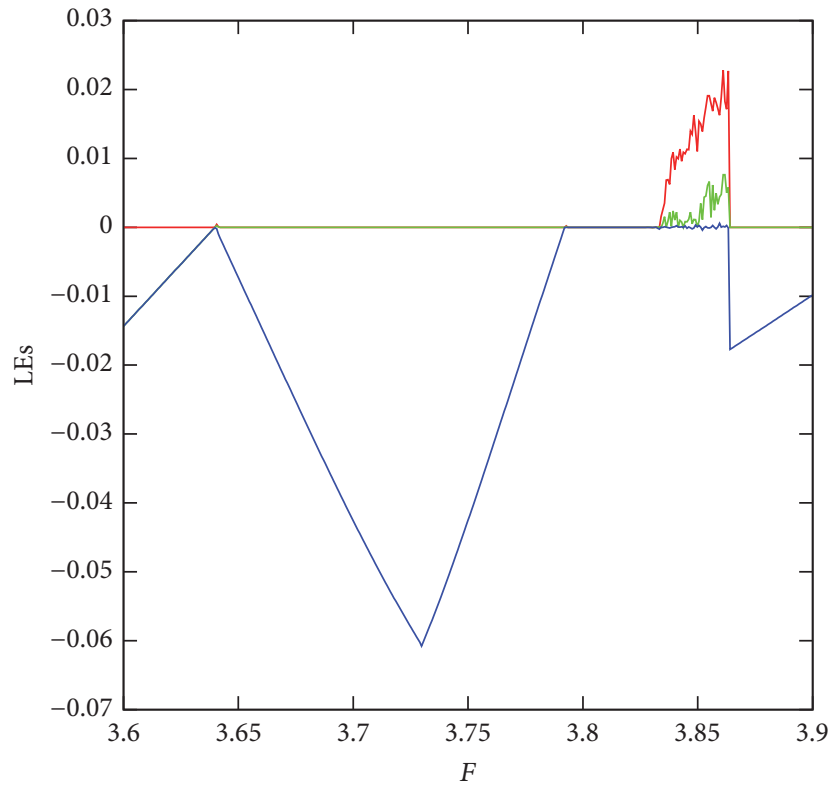

Figure 14: As Figure 3, but for dimension $n=24$. A periodic attractor bifurcates into a 2-torus attractor at $F \approx 3.639$ which in turn bifurcates into a 3-torus attractor at $F \approx 3.791$. At $F \approx 3.831$ a chaotic attractor is detected.

extremes do not become better predictable with increasing threshold.

3.4. Quasi-Periodicity for $n=24$. Figure 14 shows the bifurcation diagram of the Lorenz-96 model for dimension $n=24$. The equilibrium $x_{F}=(F, \ldots, F)$ becomes unstable at $F \approx$ 0.889 through a supercritical Hopf bifurcation. The periodic attractor remains stable until $F \approx 3.639$ where it bifurcates through a Neimark-Sacker bifurcation. The resulting 2-torus attractor remains stable until $F \approx 3.791$ and a 3-torus attractor appears. Figure 14 suggests that the 3 -torus attractor persists in a small interval of the parameter $F$ before it disappears at $F \approx 3.831$ and a chaotic attractor is detected. It is unknown which bifurcation is involved in the disappearance of the 2-torus attractor; addressing this question is left for future work. The chaotic attractor persists until $F \approx 3.864$ after which a 2-torus attractor is observed again.

The box plots in Figure 15 for $F=3.85$ show that, unlike in the cases $n=4$ and $n=7$, the singular values do not grow exponentially with the lead time $\tau$. For $F=$ $3.84,3.845,3.855,3.86$ the box plots are qualitatively similar (not shown). Errors for $\tau=0.6$ are typically larger than for $\tau=1.2$. The oscillatory behaviour weakens with increasing $\tau$. This nonmonotonic behaviour of predictability is at odds with the results in [28] in which exponential or power law growth is conjectured to be typical for chaotic systems. However, nonmonotonic dependence of predictability on lead time has also been observed in wind speed predictions produced by an operational weather forecasting system $[16$, Figure 4]. We expect that in more general systems with strong quasi-periodicity, for instance, related to diurnal or seasonal cycles, error growth rates will not follow an exponential or power law.

\section{Conclusion and Discussion}

In this paper we quantified the predictability of a specific event in a dynamical system by comparing the growth rates of errors in initial conditions that lead to this event with growth rates for all initial conditions. Numerical experiments with the Lorenz-96 model show that the predictability of large amplitudes of traveling waves is influenced by the dynamical regime of the model. In particular, we have focused on intermittency scenarios in which episodes of regular and chaotic dynamics alternate. We have shown that predictability of extremes increases near a saddle-node bifurcation of a periodic orbit but decreases near a saddle-node bifurcation of a 2-torus attractor. Finally, near the breakdown of a 3-torus attractor we have observed a nonmonotonic dependence of predictability on lead time. The results in this paper show that the predictability of extremes in dynamical systems is not universal and warrant a further in-depth investigation to unravel generic dynamical mechanisms that lead to enhanced predictability of extremes.

We have studied the predictability in the model-driven framework (borrowing the terminology of [9]). The advantage of the approach outlined in this work is that distributions of error growth rates are computed in terms of the invariant measure of the system. In this way distributions of error growth rates for vanishingly rare events can be studied. For simple classes of dynamical systems the methodology can be used to derive rigorous results on predictability and this direction will be pursued in future work. A limitation of our method, however, is the explicit need of a dynamical system and its variational equations (also referred to as a tangent linear model). The latter problem may be remedied by replacing the singular values which maximize (3) by the quotients in (2) using a small, but finite, value of $\epsilon$. Harle et al. [28] pointed out that for sufficiently small $\epsilon$ the growth rates of infinitesimal and finite-size errors behave similarly with prediction lead time.

Our work is written in the spirit of dynamical systems theory, and we have used a measure of predictability which fits into that framework. However, instead of using finitetime growth rates to measure predictability one could also use skill scores or receiver operator characteristic (ROC) curves. Such measures have the advantage that they can 


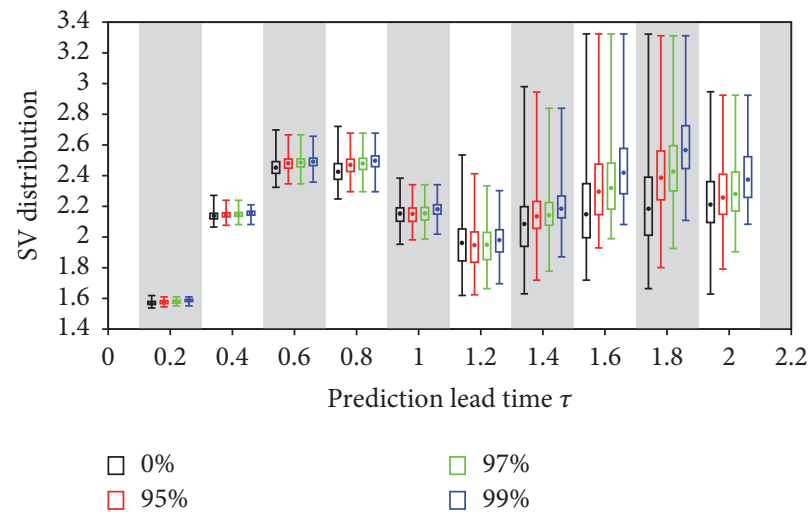

(a)

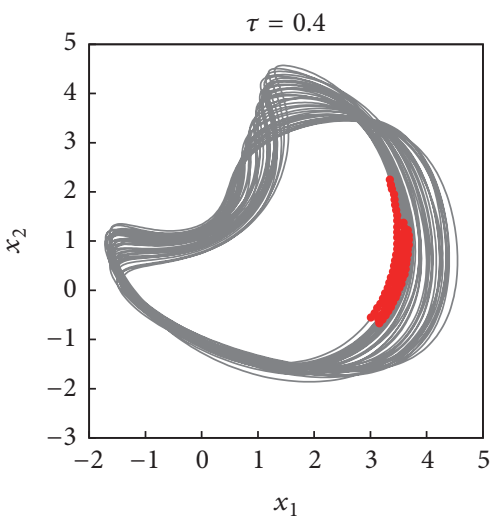

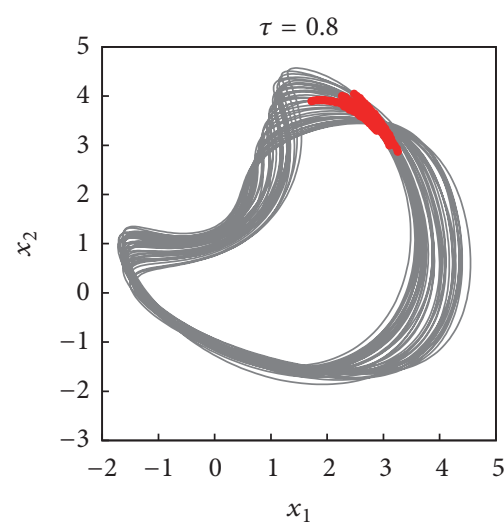

(b)

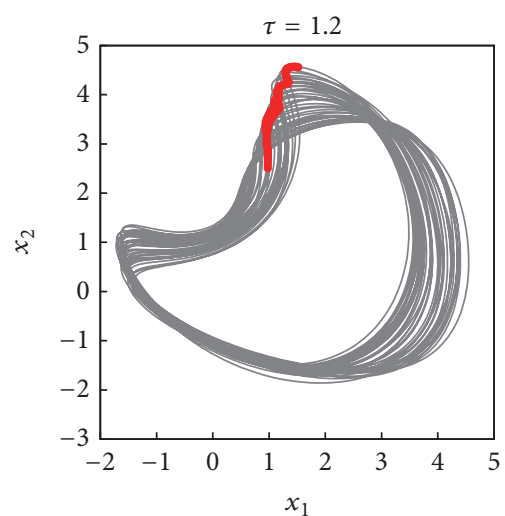

FIGURE 15: As Figure 6, but for $n=24$ and $F=3.85$.

also be used in the framework of data-driven predictions in cases where a dynamical model is not available. The numerical experiments performed with the Lorenz-84 model by Bodai [9] suggest that finite-time Lyapunov exponents do not directly correspond to ROC-based measures. This would imply that assessment of predictability also depends on which measure is being used. An interesting question for further research is how can the results of different studies using different predictability measures be reconciled and under which circumstances do different measures for predictability lead to opposite conclusions?

We conclude this paper by remarking that the phenomenon of enhanced predictability of extreme events is not limited to toy models, but it also occurs in real-world applications. Recent work [16], based on output of the operational ensemble prediction system of the UK Met Office, has revealed that wind speed extremes are in general less predictable than nonextremes, but under certain conditions which are related to the distribution of the ensemble members they are better predictable. In addition, observational work shows that large-scale flow patterns, such as the North Atlantic Oscillation, cause temporal clustering of storms [51, 52]. Hence, we foresee that the predictability of extremes will remain an active topic of research in the near future.

\section{Conflicts of Interest}

The authors declare that there are no conflicts of interest regarding the publication of this paper.

\section{References}

[1] J. Beirlant, Y. Goegebeur, J. Teugels, and J. Segers, Statistics of Extremes: Theory and Applications, John Wiley \& Sons, Berlin, Gremany, 2004.

[2] E. Castillo, Extreme Value Theory in Engineering, Statistical Modeling and Decision Science, Academic Press, Inc., Boston, Mass, USA, 1988.

[3] S. G. Coles, Introduction to Statistical Modeling of Extreme Values, Springer, New York, NY, USA, 2001.

[4] P. Embrechts, C. Klüppelberg, and T. Mikosch, Modelling Extremal Events, vol. 33 of Applications of Mathematics, Springer-Verlag, Berlin, Germany, 1997.

[5] J. Galambos, The Asymptotic Theory of Extreme Order Statistics, John Wiley \& Sons, New York, NY, USA, 1978.

[6] M. R. Leadbetter, G. Lindgren, and H. Rootzén, Extremes and Related Properties of Random Sequences and Processes, Springer Series in Statistics, Springer-Verlag, New York, NY, USA, 1983. 
[7] S. I. Resnick, Extreme Values, Regular Variation, and Point Processes, vol. 4 of Applied Probability. A Series of the Applied Probability Trust, New York, NY, USA, 1987.

[8] V. Lucarini, D. Faranda, A. de Freitas et al., Extremes and Recurrence in Dynamical Systems, John Wiley \& Sons, Inc., Hoboken, NJ, USA, 2016.

[9] T. Bodai, "Predictability of threshold exceedances in dynamical systems," Physica D. Nonlinear Phenomena, vol. 313, pp. 37-50, 2015.

[10] S. Hallerberg, E. G. Altmann, D. Holstein, and H. Kantz, "Precursors of extreme increments," Physical Review E, vol. 75, no. 1, Article ID 016706, 13 pages, 2007.

[11] S. Hallerberg and H. Kantz, "Influence of the event magnitude on the predictability of an extreme event," Physical Review E, vol. 77, article 011108, 2008.

[12] S. Hallerberg and H. Kantz, "How does the quality of a prediction depend on the magnitude of the events under study?" Nonlinear Processes in Geophysics, vol. 15, no. 2, pp. 321-331, 2008.

[13] C. Franzke, "Predictability of extreme events in a nonlinear stochastic-dynamical model," Physical Review E - Statistical, Nonlinear, and Soft Matter Physics, vol. 85, no. 3, Article ID 031134, 2012.

[14] C. L. E. Franzke, "Extremes in dynamic-stochastic systems," Chaos, vol. 27, no. 1, Article ID 012101, 2017.

[15] A. E. Sterk, M. P. Holland, P. Rabassa, H. W. Broer, and R. Vitolo, "Predictability of extreme values in geophysical models," Nonlinear Processes in Geophysics, vol. 19, no. 5, pp. 529-539, 2012.

[16] A. E. Sterk, D. B. Stephenson, M. P. Holland, and K. R. Mylne, "On the predictability of extremes: Does the butterfly effect ever decrease?” Quarterly Journal of the Royal Meteorological Society, vol. 142, no. 694, pp. 58-64, 2016.

[17] D. B. Stephenson, B. Casati, C. A. T. Ferro, and C. A. Wilson, "The extreme dependency score: A non-vanishing measure for forecasts of rare events," Meteorological Applications, vol. 15, no. 1, pp. 41-50, 2008.

[18] C. E. Leith, "Numerical simulation of the earths atmosphere," in Methods in Computational Physics, B. Alder, Fernbach. S., and M. Rotenberg, Eds., pp. 1-28, Academic Press, 1965.

[19] Y. Mintz, "Very long term global integration of the primitive equations of atmospheric motion," Tech. Rep. 94, 1964.

[20] J. Smagorinsky, "General circulation experiments with the primitive equations: I. The basic equations," Monthly Weather Review, vol. 91, pp. 99-164, 1963.

[21] V. I. Oseledec, "A multiplication ergodic theorem. Lyap unov charactoristic numbers for dynamicc system," translation of Moscow Mathematical Society, vol. 19, pp. 179-210, 1968.

[22] C. Froeschlé and E. Lega, "On the structure of symplectic mappings. The fast Lyapunov indicator: a very sensitive tool," Celestial Mechanics \& Dynamical Astronomy. An International Journal of Space Dynamics, vol. 78, no. 1-4, pp. 167-195, 2000.

[23] C. Froeschlé, E. Lega, and R. Gonczi, "Fast Lyapunov indicators. application to asteroidal motion," Celestial Mechanics \& Dynamical Astronomy, vol. 67, no. 1, pp. 41-62, 1997.

[24] F. Panichi, L. Ciotti, and G. Turchetti, "Fidelity and reversibility in the restricted three body problem," Communications in Nonlinear Science and Numerical Simulation, vol. 35, pp. 53-68, 2016.

[25] H. D. Abarbanel, R. Brown, and M. B. Kennel, "Variation of Lyapunov exponents on a strange attractor," Journal of Nonlinear Science, vol. 1, no. 2, pp. 175-199, 1991.
[26] B. A. Bailey, S. Ellner, and D. W. Nychka, "Chaos with confidence: asymptotics and applications of local Lyapunov exponents," Fields Institute Communications, vol. 11, pp. 115-133, 1997.

[27] H. Fujisaka, "Statistical dynamics generated by fluctuations of local Lyapunov exponents," Progress of Theoretical Physics, vol. 70, no. 5, pp. 1264-1275, 1983.

[28] M. Harle, F. Kwasniok, and U. Feudel, "Growth of finite errors in ensemble prediction," Nonlinear Processes in Geophysics, vol. 13, no. 2, pp. 167-176, 2006.

[29] J. M. Nese, "Quantifying local predictability in phase space," Physica D. Nonlinear Phenomena, vol. 35, no. 1-2, pp. 237-250, 1989.

[30] C. Ziehmann, L. A. Smith, and J. Kurths, "The bootstrap and Lyapunov exponents in deterministic chaos," Physica D. Nonlinear Phenomena, vol. 126, no. 1-2, pp. 49-59, 1999.

[31] C. Ziehmann, L. A. Smith, and J. Kurths, "Localized Lyapunov exponents and the prediction of predictability," Physics Letters. A, vol. 271, no. 4, pp. 237-251, 2000.

[32] R. Benzi and G. F. Carnevale, "A possible measure of local predictability," Journal of the Atmospheric Sciences, vol. 46, no. 23, pp. 3595-3598, 1989.

[33] S. Datta and R. Ramaswamy, "Non-Gaussian fluctuations of local Lyapunov exponents at intermittency," Journal of Statistical Physics, vol. 113, no. 1-2, pp. 283-295, 2003.

[34] A. Prasad and R. Ramaswamy, "Characteristic distributions of finite-time Lyapunov exponents," Physical Review E - Statistical Physics, Plasmas, Fluids, and Related Interdisciplinary Topics, vol. 60, no. 3, pp. 2761-2766, 1999.

[35] E. N. Lorenz, "Predictability-a problem partly solved," in Predictability of Weather and Climate, T. N. Palmer and R. Hagedorn, Eds., pp. 40-58, Cambridge University Press, 2006.

[36] D. L. van Kekem and A. E. Sterk, "Travelling waves and their bifurcations in the Lorenz-96 model," https://arxiv.org/ abs/1704.05442.

[37] H. Broer, H. Dijkstra, C. Simó, A. Sterk, and R. Vitolo, “The dynamics of a low-order model for the Atlantic multidecadal oscillation," Discrete and Continuous Dynamical Systems. Series B. A Journal Bridging Mathematics and Sciences, vol. 16, no. 1, pp. 73-107, 2011.

[38] A. E. Sterk, R. Vitolo, H. W. Broer, C. Simó, and H. A. Dijkstra, "New nonlinear mechanisms of midlatitude atmospheric lowfrequency variability," Physica D: Nonlinear Phenomena, vol. 239, no. 10, pp. 702-718, 2010.

[39] H. W. Broer, F. Dumortier, S. J. van Strien, and F. Takens, "Structures in dynamics: finite dimensional deterministic studies," in Studies in Mathematical Physics, vol. 2, North-Holland Publishing Co., Amsterdam, Holland, 1991.

[40] H. Broer and F. Takens, "Dynamical systems and chaos," in Applied Mathematical Sciences, vol. 172, Springer, New York, NY, USA, 2011.

[41] S. H. Friedberg, A. J. Insel, and L. E. Spence, Linear Algebra, Pearson, 4th edition, 2003.

[42] E. Ott, B. R. Hunt, I. Szunyogh et al., "A local ensemble Kalman filter for atmospheric data assimilation," Tellus, Series A: Dynamic Meteorology and Oceanography, vol. 56, no. 5, pp. 415-428, 2004.

[43] A. Trevisan and L. Palatella, "On the kalman filter error covariance collapse into the unstable subspace," Nonlinear Processes in Geophysics, vol. 18, no. 2, pp. 243-250, 2011. 
[44] D. Crommelin and E. Vanmden-Eijden, "Subgrid-scale parameterization with conditional markov chains," Journal of the Atmospheric Sciences, vol. 65, no. 8, pp. 2661-2675, 2008.

[45] G. Gallavotti and V. Lucarini, "Equivalence of non-equilibrium ensembles and representation of friction in turbulent flows: the Lorenz 96 model," Journal of Statistical Physics, vol. 156, no. 6, pp. 1027-1065, 2014.

[46] V. Lucarini and S. Sarno, "A statistical mechanical approach for the computation of the climatic response to general forcings," Nonlinear Processes in Geophysics, vol. 18, no. 1, pp. 7-28, 2011.

[47] D. Pazó, I. G. Szendro, J. M. López, and M. A. Rodríguez, "Structure of characteristic Lyapunov vectors in spatiotemporal chaos," Physical Review E - Statistical, Nonlinear, and Soft Matter Physics, vol. 78, no. 1, Article ID 016209, 2008.

[48] M. P. Holland, R. Vitolo, P. Rabassa, A. E. Sterk, and H. W. Broer, "Extreme value laws in dynamical systems under physical observables," Physica D: Nonlinear Phenomena, vol. 241, no. 5, pp. 497-513, 2012.

[49] Y. Pomeau and P. Manneville, "Intermittent transition to turbulence in dissipative dynamical systems," Communications in Mathematical Physics, vol. 74, no. 2, pp. 189-197, 1980.

[50] H. W. Broer, G. B. Huitema, F. Takens, and B. L. Braaksma, "Unfoldings and bifurcations of quasi-periodic tori," Memoirs of the American Mathematical Society, vol. 83, no. 421, 1990.

[51] P. J. Mailier, D. B. Stephenson, C. A. T. Ferro, and K. I. Hodges, "Serial clustering of extratropical cyclones," Monthly Weather Review, vol. 134, no. 8, pp. 2224-2240, 2006.

[52] R. Vitolo, D. B. Stephenson, L. M. Cook, and K. MitchellWallace, "Serial clustering of intense European storms," Meteorologische Zeitschrift, vol. 18, no. 4, pp. 411-424, 2009. 


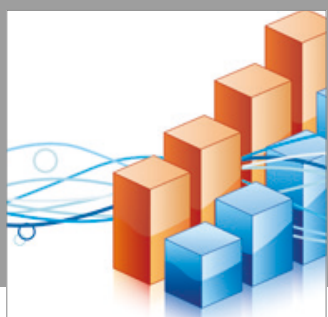

Advances in

Operations Research

vatersals

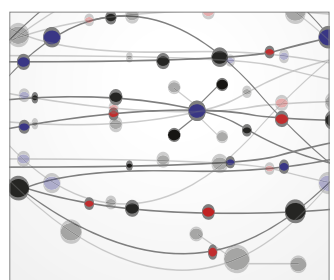

\section{The Scientific} World Journal
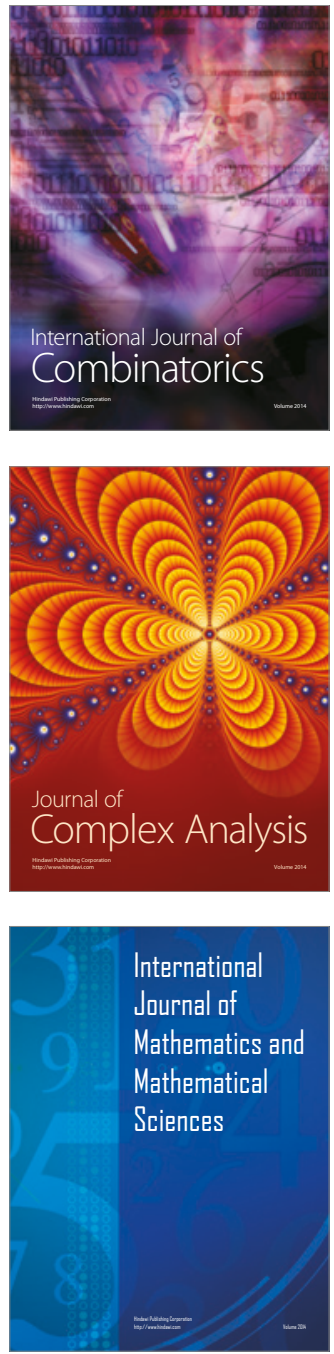
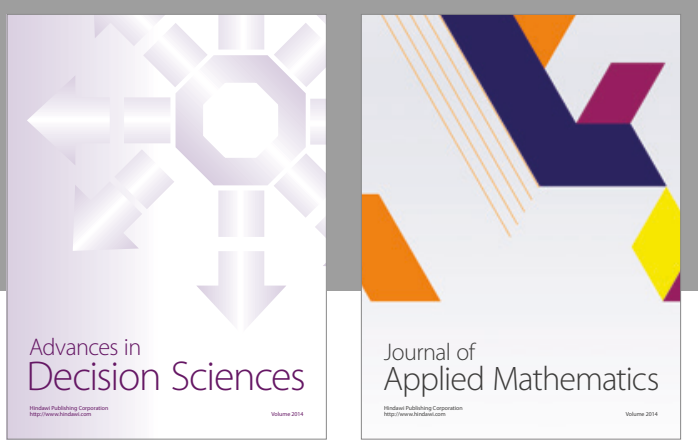

Algebra

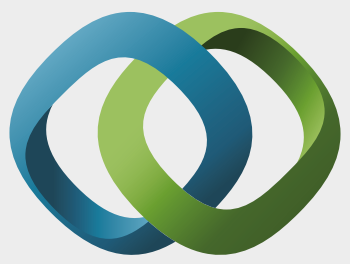

\section{Hindawi}

Submit your manuscripts at

https://www.hindawi.com
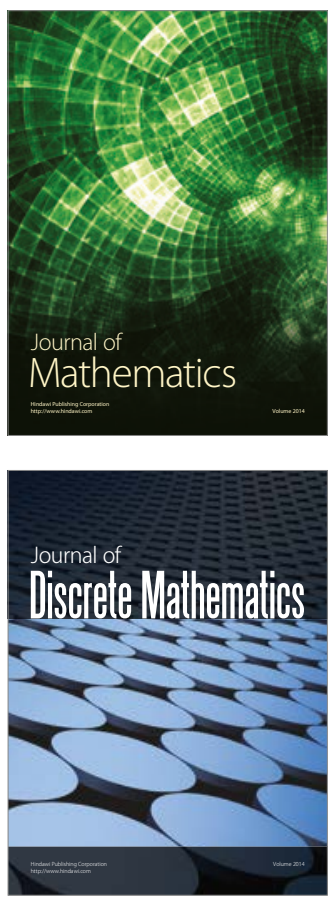

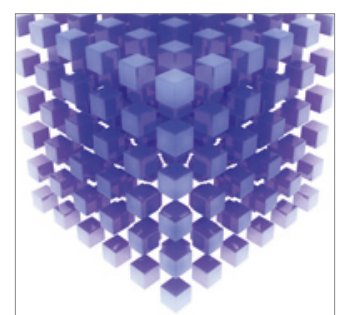

Mathematical Problems in Engineering
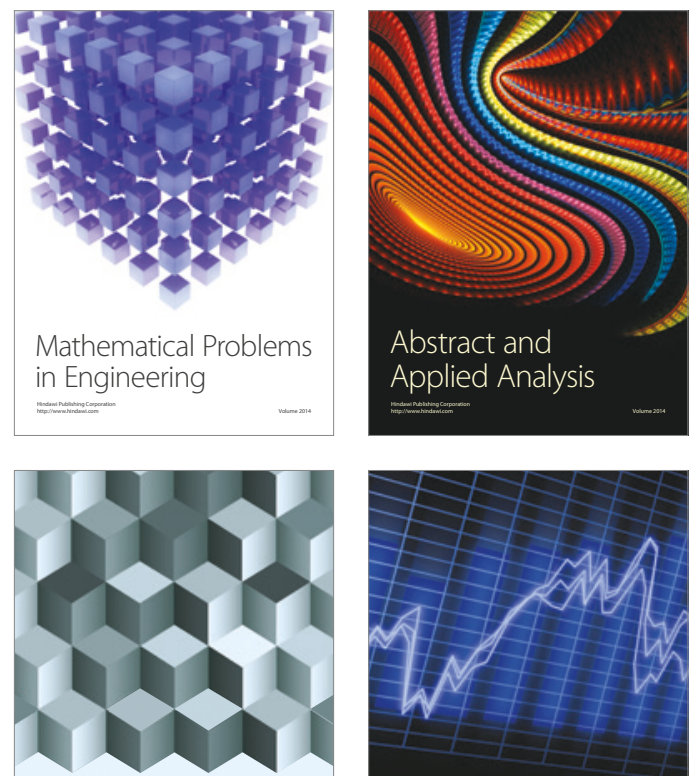

Journal of

Function Spaces

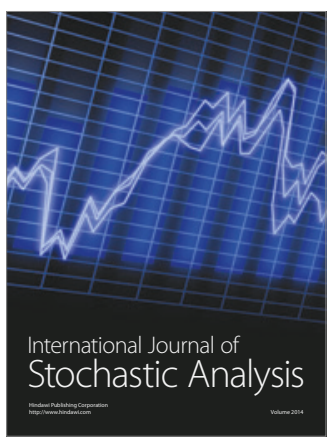

Probability and Statistics
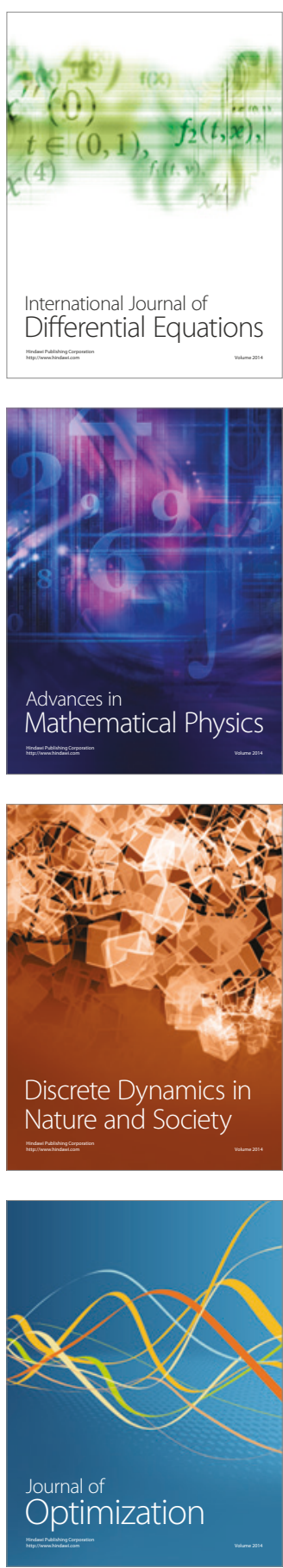